\title{
EvoBot: An Open-Source, Modular Liquid Handling Robot for Nurturing Microbial Fuel Cells
}

\author{
A. Faíña ${ }^{1,3}$, F. Nejatimoharrami ${ }^{1}$, K. Stoy ${ }^{1}$, P. Theodosiou ${ }^{2}$, B. Taylor ${ }^{2}$ and I. Ieropoulos ${ }^{2}$ \\ ${ }^{1}$ Robots, Evolution, and Art Lab (REAL), IT University of Copenhagen, Denmark \\ ${ }^{2}$ Bristol BioEnergy Centre, Bristol Robotics Laboratory, University of the West of England, Bristol, UK \\ ${ }^{3}$ Corresponding author: anfv@itu.dk
}

\begin{abstract}
Liquid handling robots are rarely used in the domain of artificial life. In this field, transitory behaviours of nonequilibrium man-made systems are studied and need an automatic monitoring and logging of results. In addition, artificial life experiments are dynamic with frequent changes, which makes it difficult to apply conventional liquid handling robots as they are designed to automate a pre-defined task. In order to address these issues, we have developed an open source liquid handling robot, EvoBot. It uses a modular approach, which gives us the possibility to reconfigure the robot for different experiments and make it possible for users to add functionality by just developing a function specific module. In addition, it provides sensors and extra functionality for monitoring an experiment, which allows researchers to perform interactive experiments with the aim of prolonging non-equilibrium conditions. In this paper, we describe the modular design of EvoBot, document its performance, and provide a novel example of an interactive experiment in artificial life, where the robot nurtures a microbial fuel cell based on its voltage output.
\end{abstract}

\section{Introduction}

Liquid handling robots are often employed in chemical and biochemical laboratories in order to automate repetitive tasks (see (Kong et al. 2012) for a useful overview of liquid handling robots for lab automation). The benefit of this is typically an increase in reliability, throughput and precision when compared to manual liquid handling coupled with a reduction in labour cost. However, liquid handling robots are rarely used in the domain of artificial life.

There are two types of challenges that make existing liquid handling robots inappropriate for experiments in artificial life. The first relates to the functionality needed from the robot to be useful in artificial life research. In artificial life we are mostly interested in the initial condition of an experiment and how it develops but not typically in the end result, because the end result is equilibrium which in the context of artificial life corresponds to death. This means that not only should the robot prepare experiments, but also employ automatic monitoring and logging of results. Furthermore, it would be desirable if the liquid handling robot, based on this monitoring, can interact with the experiment to extend its life-time. Solving this challenge has been the basis for the results of (Gutierrez, 2012; Gutierrez et al., 2014; Hanczyc et al. 2015, Müller et al., 2015), who all made an ad-hoc liquid handling robot for specific experiments in artificial chemical life.

The second type of challenge, which is the focus of our work, is the practical challenge of employing liquid handling robots in artificial life research. Artificial life research is often performed in small, government funded labs and as such the cost of acquisition is a limiting factor. Artificial life experiments are not static, but develop in response to the insights obtained from the experiments and this makes it difficult to apply conventional liquid handling robots as reprogramming and reconfiguration is often tedious and timeconsuming if at all possible for the end user. Furthermore, conventional liquid handling robots are often only setup to perform one specific task, thus making it difficult to modify with functionalities required for new types of experiments. If we could provide solutions to these challenges, artificial life research could also benefit from the advantages of automation namely increased reliability, throughput, precision and reduced cost. Turning these challenges around we are looking for liquid handling robot technology that has the following characteristics:

- Reconfigurable

- Versatile

- Low cost

- Extendable

In order to address these challenges, we propose the use of a modular design borrowing a significant number of advantages from modular robots(Yim et al. 2007). A modular design allows a non-expert user to reconfigure the robot for different experiments by swapping in and out modules. Expert knowledge is only required when designing modules with new functionalities. A modular approach also increases the versatility of liquid handling robots because they can easily be reconfigured for many different types of experiment 

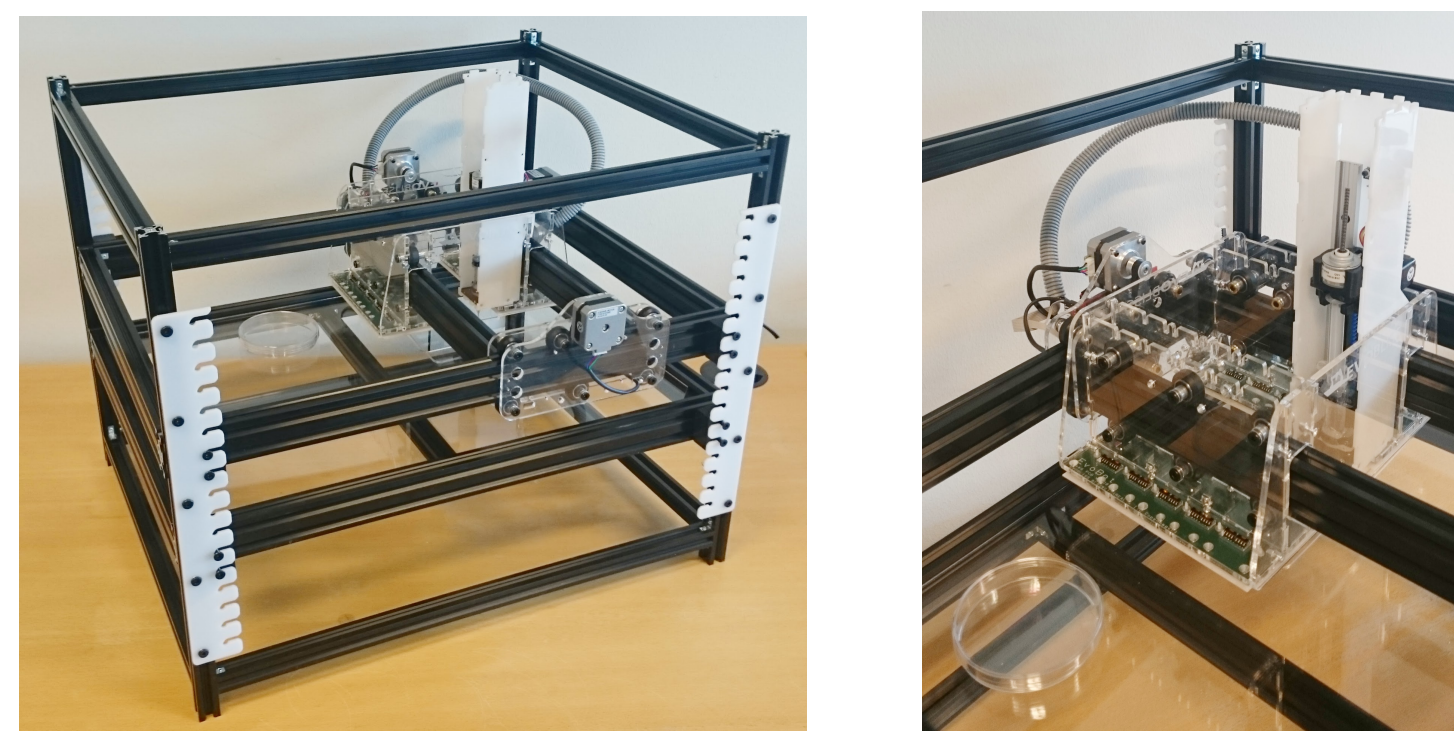

Figure 1: The EvoBot liquid handling robot (left) and a close-up of the movable head equipped with one syringe module.

during their life-time. We have made EvoBot open-source, which reduces the cost of acquisition and also allows researchers to build on our reliable platform and extend it with modules that have the necessary functionality for their specific experimen 11

In this paper we present the modular design of the liquid handling robot, EvoBot, and carefully document its performance to make it possible for potential users to evaluate how appropriate it is for their experiment. We have already demonstrated its usefulness in artificial chemical life $(\mathrm{Ne}-$ jatimoharrami et al. 2016), but here we further evaluate its use in a new application domain, nurturing microbial fuel cells (MFCs). MFCs are devices where microbes convert organic matter directly into electricity. MFCs have demonstrated their utility as a basis for building robots with an artificial metabolism (Ieropoulos et al. 2010).

The conclusion is that EvoBot due to its versatility, extendability, and low cost was successfully implemented in the new application domain of nurturing microbial fuel cells with very limited modification. It is also demonstrated that using the robot for maintaining microbial fuel cells, compared to manually nurturing them, has numerous advantages, and thus in time may provide insights that can help researchers develop more efficient microbial fuel cells.

\section{EvoBot Design Principles}

As outlined above, a key goal of the EvoBot design was to develop a robotic platform, which can be configured for a

\footnotetext{
${ }^{1}$ Source code and design files can be found in Git repositories, https://bitbucket.org/afaina/ evobliss-software and https://bitbucket.org/ afaina/evobliss-hardware respectively.
}

wide range of experiments without the involvement of an expert user. In order to achieve this, we modularised the design building on research in the field of modular robotics (Yim et al. 2007). A modular approach allows us to encapsulate complexity while providing a simple mechatronics plug'n'play interface to the system.

EvoBot (see Figure 1) consists of one structural frame and three types of layers, which in the default configuration are organised as follows: the top layer carries actuation, the middle layer is the experimental section, and the bottom layer is an observation platform. However, this default layout can easily be amended, e.g. several experimental layers can be introduced if a cascading experiment is under investigation. These three layers can easily be moved up and down in the frame. Functionality is provided in the form of modules, which allow functionality to be added incrementally in the form of new modules.

In order to create a high quality and cheap platform, we built the robot from off-the-shelf components and, where possible, used components used by the open-source 3D printer community and therefore readily available. For instance, we used Nema17 motors for actuation and Arduino based electronics for control of the robot. Another key principle was to favour laser-cut acrylics over 3D printing. The reasons being lower production time and high-quality of the produced components in comparison with the elements produced through 3D-printing. However, we did use 3D printed components outside the core mechanical structure, primarily inside the syringe module (described later) due to the geometrical flexibility afforded by 3D printers. 


\section{EvoBot Implementation}

In the following section, we will provide an overview of the hardware (mechanics \& electronics), and software of EvoBot.

\section{Mechanics}

First, we will describe the layers of EvoBot in more detail followed by the implementation of the modules.

Frame The frame is made of aluminium profiles and it provides the physical support for the layers. They can be attached to the frame at specific heights every $20 \mathrm{~mm}$. Additionally, it allows levelling the robot with four adjustable feet. The frame measures $600 \times 400 \times 600 \mathrm{~mm}$ but it can easily be extended.

Layers The EvoBot platform is organised into three types of layer: actuation, experiment, and observation layers.

The actuation layer contains a head, which can move in the horizontal plane using two belt and pinion mechanisms and two stepper motors. Up to 11 modules can be mounted on this head to provide different functionalities. At this point, only an actuated syringe module and a heavy payload module have been implemented, but various actuators and sensor modules are envisaged, e.g. temperature sensor, $\mathrm{pH}$ sensor, gripper for manipulation of dishes, extruder for printing reaction vessels, etc.

The experimental layer is essentially just a frame with a glass plate where vessels can be organised as required by the specific experiment. There is a hole in one corner, where vessels can be moved and dropped for automatic disposal, and together with a Petri dish dispenser system under development, a large number of experiments can be done in sequence. However, we have found that for now it is enough to clean reaction vessels by going through three water wash cycles, where vessels repeatedly are filled with water and emptied using the syringe modules. This makes the dispenser functionality less critical for long term operation. However, for more sensitive experiments the vessels and also syringe tips may have to be replaced on a per experiment basis.

The observation layer is essentially the same as the actuation layer except that modules cannot interact physically with the experiments above, because they are shielded by the glass plate. This limits the useful modules for this layer to modules that do not directly manipulate the experiments. Currently, a static webcam is used in the observation layer to monitor the experiment and provide feedback to the robot. However, in the longer term thermal imaging, magnetic stirring, liquid effluent sampling or the like could be integrated in modules for the observation layer.

Syringe and Heavy Payload Module The syringe module can be seen in Figure 2 a-b has two degrees of freedom. It has a linear stepper motor (a stepper motor with a lead screw

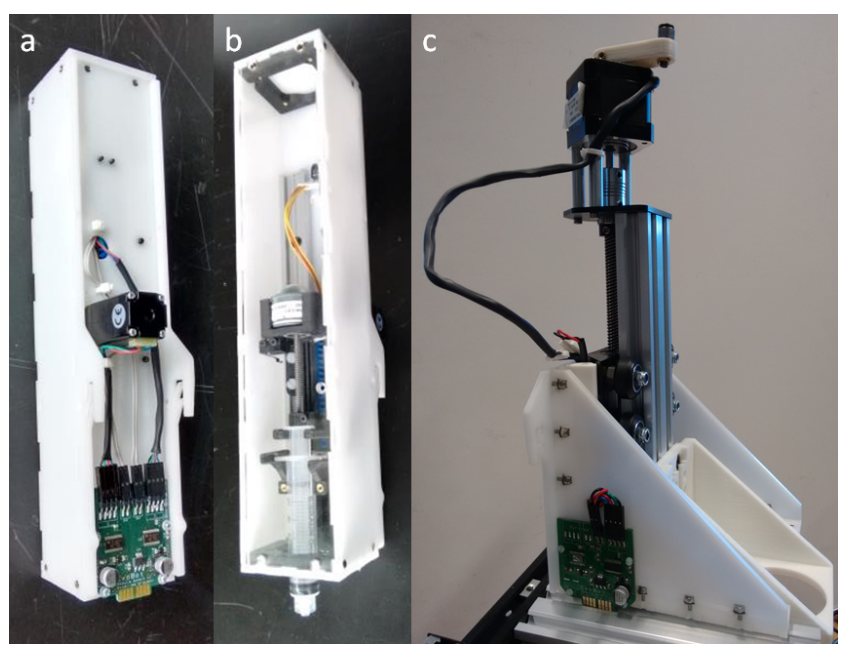

Figure 2: Syringe module (a, b) and heavy payload module (c).

and an internal nut) for moving the plunger up and down and a rack and pinion mechanism with another stepper motor for moving the syringe up and down. Syringes up to $20 \mathrm{ml}$ can be used and they can easily be replaced giving the user the opportunity to use the syringe that matches the experimental requirements.

The heavy payload module is designed to hold and move up and down big and heavy parts. A lead screw mechanism is used to hold the payload even if its motor is switched off. A Nema 17 stepper motor moves the payload but it can also be manually operated with a crank. Its stroke is $80 \mathrm{~mm}$ and the maximum speed is $8 \mathrm{~mm} / \mathrm{s}$. This module is shown in Figure 2 $\mathrm{c}$ and its end effector is designed to hold an OCT scanner, but it can easily be modified to hold other devices.

More modules are under development including a module with a gripper, and a module to measure $\mathrm{pH}$. However, an EvoBot with one syringe is enough to perform useful experiments in artificial chemical life as demonstrated in (Hanczyc et al. 2015; Gutierrez et al., 2014).

\section{Electronics}

The core of the electronics of EvoBot is based on electronics used in the open-source 3D printer community. Specifically, we use the Arduino MEGA 2560 R3 with the shield RAMPS v1.4. This provides us with a mature and cheap electronics platform to build on, but perhaps more importantly, allows us to build on existing software for open-source 3D printers.

A key aspect in the electronics design was to keep the number of wires between components as low as possible to avoid interference with experiments, become a source of error if disconnected accidentally, and to give the robot a clean look. In order to do this, a circuit board was designed that routes power and communication to the modules on the head of the robot. This board is fairly large, but con- 
sists only of simple routing, two input/output port expanders with serial interface (I2C) and spring connectors. For the modules, a custom board was also made that contains two stepper drivers with SPI communications (L6470) for moving up and down the stepper motors of the module, see Figure 2 a. When a module is placed on the head, the spring connector touches the pads of the board of the syringe and transmits power and the SPI signals. Furthermore, the wires between the Arduino and the head are reduced using the I2C expander ports as they generate the Chip Select signals for the SPI communication. Using this approach, only a power cable and an 8-way ribbon cable with the I2C and SPI buses are necessary to manage the 22 motors of the 11 syringe modules, which can be placed on the head.

\section{Software}

The goal of the software was to provide the end-user with a simple programming interface to the robot. The software has a host side and a robot side. The host side communicates with the robot side over a serial USB connection and the robot side software is a modified version of the Marlin firmware used in open-source 3D printers.

On the host side, we have chosen Python as the implementation language as this was the language with which our collaborators have most experience and also due to its simplicity. The software is divided into a graphical user-interface for manual control of the robot and a simple application programming interface.

Robot Manual Control The purpose of the manual control graphical user interface is simply to be able to test various functions of the robot without having to resort to programming. However, the most important use of the manual control program is to learn the position of interesting objects in the robots coordination frame. For example, the user could move the robot until it is approximately in the centre of a Petri dish of interest and lower the syringe so the tip is in the vessel. This is an empirical way of defining the geometry for an experiment, and perhaps the most practical for a range of experiments.

Application Programming Interface The application programming interface is also kept as simple as possible. The interface gives the programmer access to moving the robot head, moving the syringes and plungers, and inquiring about the positions of these elements.

The application programming interface is built on top of printcore, which is an open source Python library for interacting with 3D printers and pySerial, which handles the serial communication. The printcore library, however, has been heavily reduced and is likely to be unnecessary in the near future.

At the bottom of the software stack we have implemented a basic simulation that can be swapped instead of the serial communication library. This makes it possible to debug
Table 1: Max speed and accelerations of the robot.

\begin{tabular}{lllll}
\hline & $\mathrm{X}$ & $\mathrm{Y}$ & $\begin{array}{l}\mathrm{Z} \\
\text { (Syringe) }\end{array}$ & Plunger \\
\hline $\begin{array}{l}\text { Max Speed } \\
(\mathrm{mm} / \mathrm{s})\end{array}$ & 180 & 180 & 235 & 8 \\
$\begin{array}{l}\text { Acceleration } \\
\left(\mathrm{mm} / \mathrm{s}^{2}\right)\end{array}$ & 3000 & 3000 & 235 & 4 \\
\hline
\end{tabular}

the upper layers without actually moving the physical robot. This is a feature that has saved a significant amount of development time.

Marlin-Based Firmware On the robot side we run an extended version of Marlin, a firmware used to control opensource 3D printers. This gives us a mature basis for our robot controller. The head of the actuation layer is controlled directly using the functionality Marlin provides. For the syringe or heavy payload modules, we have extended Marlin with G-code commands for interacting with them. The use of Marlin is also beneficial as it is our plan to make an extruder module, which can 3D print reaction vessels and thus this aspect of the firmware can also be put to good use.

\section{Precision and Performance}

For the reader interested in understanding if EvoBot is suitable for their experiments and to facilitate comparison between liquid handling robots, we provide below a careful investigation of the precision and performance of EvoBot.

\section{Speed}

A key parameter to perform experiments is the speed of the robot because the experiments could take a long time if the speed of the robot is low or it is not able to manage a set of tests simultaneously. With this objective in mind, the maximum speed of the robot was tested for each axis. The test consisted of movements on each axis, increasing its acceleration and maximum speed until the robot lost some steps. In other words, the maximum speed is when the positioning of the robot after several movements is not accurate anymore. Table 1 contains the results of these tests and displays that they are significantly higher than the requested accuracy by our collaborators.

\section{Positioning Performance}

The positioning performance has been evaluated based on the ISO 9283:1998. Thus, one syringe module was modified to hold a test probe, instead of a syringe. This probe was used as an end effector to touch a dial indicator, which has an accuracy of $0.01 \mathrm{~mm}$. Given the short stroke of the indicator $(25 \mathrm{~mm})$, we ran two different tests to calculate the accuracy and the repeatability of the robot on each axis. 
Table 2: Positioning accuracy for each axis of the robot.

\begin{tabular}{cccccc}
\hline & $0 \mathrm{~mm}$ & $1 \mathrm{~mm}$ & $2 \mathrm{~mm}$ & $10 \mathrm{~mm}$ & $20 \mathrm{~mm}$ \\
\hline $\begin{array}{c}\mathrm{X} \\
(\mathrm{mm})\end{array}$ & 0.04 & 0.03 & -0.04 & -0.11 & -0.17 \\
$\begin{array}{c}\mathrm{Y} \\
(\mathrm{mm})\end{array}$ & 0.01 & -0.05 & -0.08 & -0.14 & -0.18 \\
$\begin{array}{c}\mathrm{Z} \\
(\mathrm{mm})\end{array}$ & 0.10 & 0.02 & 0.09 & 0.28 & 0.35 \\
\hline
\end{tabular}

Table 3: Repeatability for each axis of the robot.

\begin{tabular}{llll}
\hline & $\mathrm{X}$ & $\mathrm{Y}$ & $\mathrm{Z}$ \\
\hline $\mathrm{RP}(\mathrm{mm})$ & 0.067 & 0.013 & 0.106 \\
\hline
\end{tabular}

Accuracy is simply defined as $A=\bar{q}-q_{t}$, where $q_{t}$ is the target position and $\bar{q}$ is the average of $\mathrm{n}$ measured coordinates. In this test, the robot was homed, and the dial indicator was placed in contact with the probe and set to zero. Then, the robot moved one axis sequentially to five different positions $(1,2,10,20$ and $0 \mathrm{~mm})$ and the real position of the probe was measured. This sequence was repeated 30 times and the accuracy for each point was calculated. The data is shown in Table 2, where we can observe that the positioning is quite reasonable for a low cost machine and there is no noticeable discrepancy. Furthermore, the slow increment of the inaccuracy when moving the robot to 10 and $20 \mathrm{~mm}$ is mainly caused by a misalignment between the axis of the indicator and the axis of the robot. This is confirmed by moving the robot to longer distances where we do not detect any appreciable error. In the future, we will employ computer vision technology to calibrate the positioning accuracy measuring the three axes at the same time.

Regarding the repeatability or precision of the robot, we ran another experiment. Thus, five points were defined all along the workspace of the robot and the robot moved the probe sequentially from one point to the next one. At the last point, the end effector touched a dial indicator to measure its coordinates. This sequence was repeated 30 times for each axis and repeatability was calculated using Equation 11. where $\mathrm{q}$ are the measurements, $\bar{q}$ is the average of these measurements, $\mathrm{n}$ the number of trials, $\mathrm{S}$ the standard deviation. Table 3 shows the repeatability for each axis. Results display very good repeatability in the three axis, around or less than $0.1 \mathrm{~mm}$.

$$
R P=3 S_{q}=3 \sqrt{\frac{\sum_{i=1}^{n}\left(q_{i}-\bar{q}\right)^{2}}{n-1}}
$$

\section{Liquid Handling Performance}

In order to measure the precision and accuracy of handling droplets, we performed various experiments with different

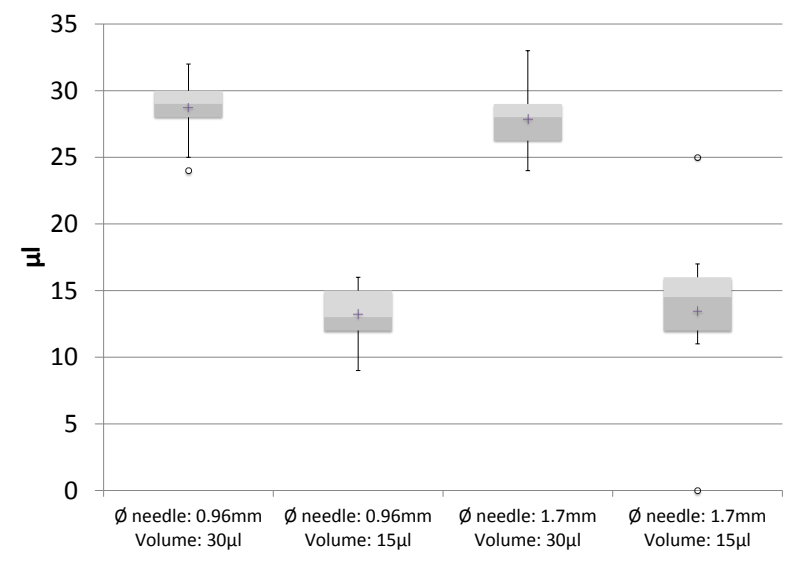

Figure 3: Boxplot comparing experiments with $100 \mu$ l syringe. The whiskers represent the lowest and highest datum still within 1.5 IQR (interquartile range) of the lower quartile or of the upper quartile, respectively.

syringe sizes, needle diameters and droplet volumes. The experiment was to absorb distilled water from a Petri dish and dispense it over a scale with $\pm 0.003 \mathrm{~g}$ precision, and therefore obtaining droplet volume by the mass-volume relation. Four experiments were performed with a professional $100 \mu \mathrm{l}$ syringe (Hamilton $710 \mathrm{LT}$ ) to handle liquid volumes of $15 \mu \mathrm{l}$ and $30 \mu \mathrm{l}$, with needle internal diameters of $0.96 \mathrm{~mm}$ and $1.7 \mathrm{~mm}$. To obtain better results in these experiments, a small quantity of air was taken into the syringe prior to absorbing water. Four other experiments were performed with a cheap disposable $5 \mathrm{ml}$ syringe (Braun Omnifix) to handle liquid volumes of $1 \mathrm{ml}$ and $0.5 \mathrm{ml}$. In these experiments, the needle internal diameters were $0.96 \mathrm{~mm}$ and $1.7 \mathrm{~mm}$. Each experiment was run 30 times.

The results of the experiments have been compared using box-plots with $100 \mu \mathrm{l}$ and $5 \mathrm{ml}$ syringes respectively, Figure 3 and 4 . Considering the error introduced by the scale, the results obtained with the $100 \mu \mathrm{l}$ syringe are quite reasonable. They are all accurate and the repeatability is good, except for the $15 \mu \mathrm{l}$ test with a needle of $1.7 \mathrm{~mm}$. This is due to surface tension, which randomly prevents the last droplet from being ejected. That amount of residual water in the tip, is taken back in during the next test, which results in an additively larger volume of water, with the fresh sample coming in. This explains the outliers of the experiment $(0$ and $25 \mu \mathrm{l}$ respectively). Regarding the experiments with 1 and $0.5 \mathrm{ml}$, results show some inaccuracy, but an acceptable repeatability. Nevertheless, we could calibrate the robot to increase the accuracy while keeping costs low. In addition, (again due to surface tension) the last droplet is lost in almost all the tests, which decreases the repeatability. Again, this effect is more noticeable for the needle with the $1.7 \mathrm{~mm}$ diameter. We will study how to avoid this negative effect 


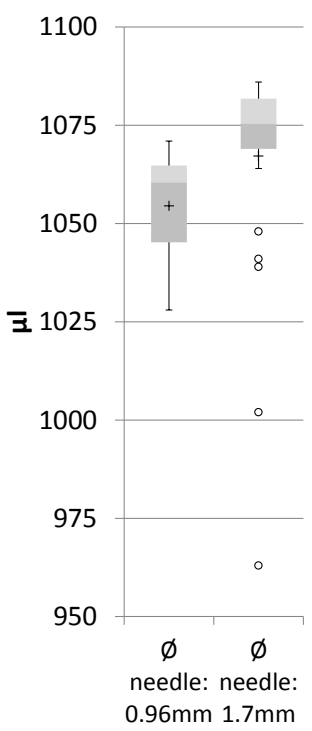

(a) $1 \mathrm{ml}$ experiment

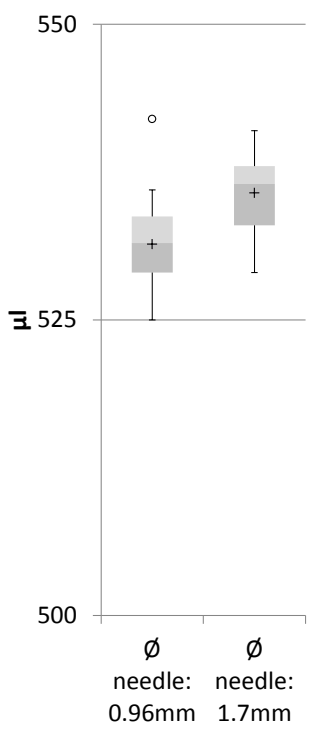

(b) $0.5 \mathrm{ml}$ experiment
Figure 4: Boxplot comparing experiments with $5 \mathrm{ml}$ syringe. The whiskers represent the lowest and highest datum still within 1.5 IQR of the lower quartile or of the upper quartile, respectively.

in future work. Some alternatives could be to increase the speed of the plunger while dispensing the liquid, to generate a small vibration when moving up the syringe quickly or to use pipettes with low surface tension.

\section{Interactive Feeding of Microbial Fuel Cells}

In this experiment we document how we applied EvoBot to the task of maintaining microbial fuel cells (MFCs). The microbial metabolism, utilises the carbon energy source within the anode chamber, which eventually depletes the carbon content and results in decreasing the voltage output from the MFC; in other words, as the fuel runs out, the voltage decreases. Thus, the robot feeds the MFC with more organic material when the voltage is below a threshold. And as a result of the interaction, the experiment is prolonged.

In order to analyse the advantages of using a liquid handling robot, two parallel microbial fuel cell experiments were performed using identical materials and methods with the only difference being that one of them was carried out manually, as a replica (control) experiment, and the other one was carried out by the EvoBot platform (Figure 5). In the case of the robot experiment, the voltage is sampled every minute, the MFCs are hydrated every four hours and only fed if the voltage is below the specified threshold. In contrast, the voltage of the replica (on the bench) was sampled every three minutes, and the MFCs were hydrated twice a day - morning and afternoon - and fed once every morning.

\section{Microbial Fuel Cell Structure}

Nine small-scale, 3D printed from Nanocure ${ }^{\circledR}$ resin, opento-air cathode MFCs were used in these experiments. The volume of each of the fuel cell anode chambers was 6.25 $\mathrm{mL}$, and the anode and cathode chambers were separated by a single sheet of activated cation exchange membrane, CMI7000S (Membranes International Inc., Ringwood, USA). Two rubber gaskets, one for each half-cell, sandwiched the membrane, and ensured watertight sealing, after the two chambers were bolted together using stainless steel studding and nuts.

\section{Electrode Material (Anode and Cathode)}

Untreated (catalyst free) carbon fibre veil, with $30 \mathrm{~g} / \mathrm{m}^{2}$ carbon loading (PMF Composites, Dorset, U.K.) was used as anode electrode with a total surface area of $168 \mathrm{~cm}^{2}$. The anode electrode was folded down 5 times, until the projected (exposed) surface area was $5.25 \mathrm{~cm}^{2}$ and could therefore fit into the anodic chamber $(18 \mathrm{~mm} \times 28 \mathrm{~mm})$. The cathode electrode was made of two layers, a gas diffusion layer (GDL) and a Micro-Porous Layer (MPL). The GDL was a single sheet of carbon veil coated with 30\% Polytetrafluoroethylene (PTFE) (Sigma Aldrich, UK). Once GDL cured, activated carbon paste was applied on top to form a thick MPL ( $1 \mathrm{~mm})$. The activated carbon paste was a mixture of activated carbon powder (G.Baldwins \& Co., London, U.K.) blended with PTFE in a 4:1 ratio and deionised water (120 $\mathrm{mL}$ ). The activated carbon paste was then hot pressed, using a household iron (Gajda et al., 2015), and subsequently heated for 15 minutes to $200^{\circ} \mathrm{C}$ to allow MPL liquefaction.

\section{Inoculation}

For the MFC experiment on the EvoBot platform, the inoculation (i.e. introduction of live microorganisms in a sterile MFC) was done using the anolyte from already established MFCs. This was activated sludge fed with carbon sources such as tryptone yeast-extract (TYE) over a period of months, which had been sieved to remove large particles $(>1 \mathrm{~mm})$ so as to prevent blockage of the syringe needle on Evobot. For the replica bench experiment, neat activated sludge supplied by the Wessex Water Scientific Laboratory (Saltford, UK) was used as the initial inoculum. All MFCs in both experiments were kept under a fixed load of $3.9 \mathrm{k} \Omega$, for the whole duration.

\section{Experimental software setup}

Each MFC was individually connected to a separate channel on the Picolog data acquisition unit (ADC-24, Pico Technology, Cambridgeshire, UK) which was then connected to a PC, so that the DC voltage output of each unit could be continuously recorded. A set of MFC feed functions was created in LabView (National Instruments Corporation [UK] Ltd, Berkshire, UK), which sampled the Picolog DLL file every 60 seconds for the MFC voltage reading. A threshold 


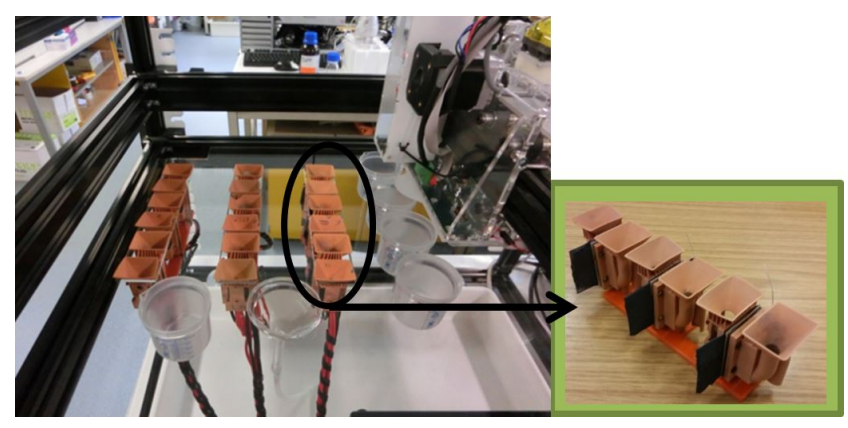

Figure 5: Interactive Feeding of Microbial Fuel Cells. Nine individually connected MFCs were operated with Evobot platform. The experiment took place on the experiment layer of the robot. The replica bench experiment was setup in exactly the same manner.

limit for each MFC was set in LabView where if the voltage dropped below this threshold, then a Python script would activate to move the head of the robot over the food source, draw $3 \mathrm{~mL}$ of substrate (carbon fuel) and then inject this into the MFC.

After the feed substrate has been deposited into the anode, the syringe module would go through a wash cycle where $3.5 \mathrm{~mL}$ of $70 \%$ ethanol was drawn into the syringe and disposed of down a waste tube on the Evobot platform, followed by the taking of $3.5 \mathrm{~mL}$ of sterile distilled $\mathrm{H}_{2} \mathrm{O}$ into the syringe, before disposing this down a waste tube and then returning the robot head to the home position. At the home position, the feed function paused for 60 minutes to allow stabilisation of the MFC and for the voltage to increase above the threshold.

A cathode hydration cycle was also incorporated in a separate function in LabView, which activated a Python script every 4 hours. This script moved the robot head over the position of each MFC and deposited $3.12 \mathrm{~mL}$ of deionised water into the cathode chamber before returning to the home position.

Although for the Evobot experiments, the 'wash cycle' with ethanol and deionised water was deemed necessary, in order to avoid the cross-contamination between the different carbon-energy sources (acetate, lactate and cellulose), this was not necessary for the replica bench experiment, which was carried out manually.

\section{Results}

The experiment lasted approximately 8 weeks for the Evobot experiment and 4 weeks for the replica and the comparisons between the experiments are made for the same 4 week period. The power output of one MFC fed by EvoBot and the feeding events are shown in Figure 6

A significant performance difference was found between the replica experiment and the EvBbot experiment; the replica experiment (data not shown) showed higher power

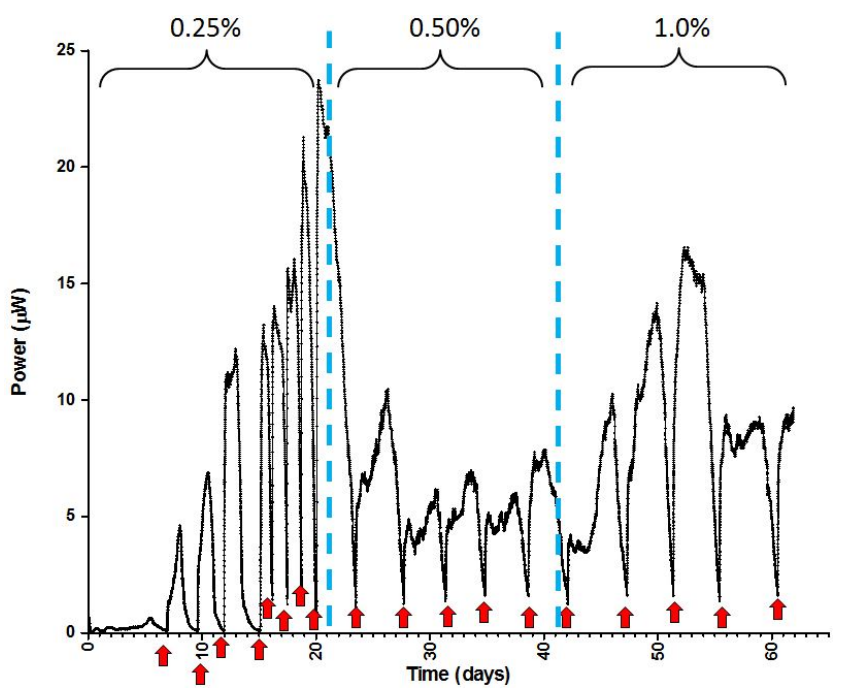

Figure 6: Power output from one of the MFCs (MFC2) fed by the Evobot with sodium acetate. The red arrows indicate the points where the voltage output of the MFC dropped below the $80 \mathrm{mV}$ threshold, which was the trigger for feeding the MFC. The dotted lines indicate the periods during which three different concentrations of sodium acetate were tested.

output levels from the MFCs, compared to those from the MFCs on EvoBot. This could have been due to the wash cycle with ethanol, which would have inevitably left residual ethanol in the syringe during the course of the experiment - the replica experiment did not require a cleaning cycle. Also, the sieving of sludge for the inoculation of the MFCs may have well resulted in a less enriched inoculum, and this was done to prevent the syringe needle from blocking - again, this was not an issue for the parallel bench experiment.

Nevertheless, having automated feeding pulses which were dictated by the voltage threshold, the behaviour of a MFC could more closely be monitored "day or night" in a way that would otherwise require an operator to be continuously present. In addition, the automated hydration cycle was advantageous, since it helped us identify empirically the aqueous $\mathrm{O}_{2}$ saturation levels for the oxygenreduction-reaction (ORR) that is necessary for the open-toair-cathodes. In other words, beyond this 'performance saturation' point, the addition of more water did not result in an increased MFC performance. This is shown in Figure 7. which illustrates the behaviour of the acetate fed MFC, from days 31-34 (data taken from Figure 6). The fluctuating electrical output is in the response to the cathode hydration, however as can be seen, the overall performance during that feeding cycle, remains the same (on average). 


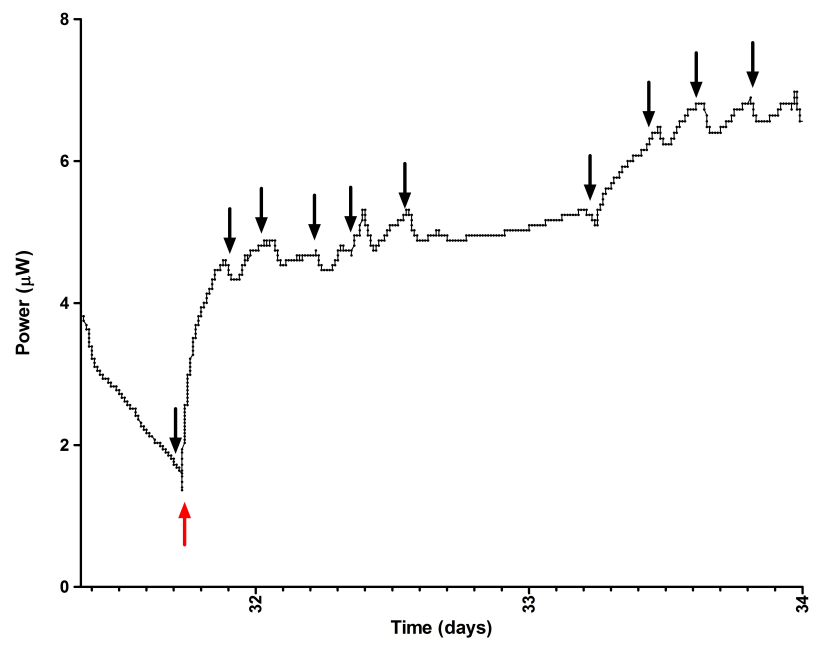

Figure 7: Power output from the same MFC2, following cathode hydrations by Evobot, during one feeding cycle between days 31-34 of the experiment. Black arrows indicate points of EvoBot hydration; the red arrow indicates a point of feeding.

\section{Future Work}

We are currently working to address the issues caused by the 'wash cycle'. A simple option would be to increase the number of times that the syringe is washed with distilled water, but we are also looking into other different techniques to avoid cross contamination. First, a dispensing module is under development, which uses external pumps to provide pure reagents or solutions. Thus, different organic materials could be used to feed the MFCs without having to use the same syringe. Additionally, we are also starting to use disposable pipette tips, but they have to be placed in the syringe manually. We hope to automate the change of these pipette tips in the future.

Regarding the interactive experiments, they are now based on the voltage of the MFCs. However, we are planning to extend the robot with sensing modules for measuring more parameters. As an example, we are developing a pH module, which will allow us to control the $\mathrm{pH}$ of the MFCs by adding acid or alkaline solutions. Our intention is that new interactive experiments will allow us to pose different scientific questions, possess novel data never recorded before and help us develop better MFCs.

\section{Conclusions}

The EvoBot robot is an open-source, modular liquid handling robot. Our design focuses on high quality, relying on open-source components and software, and being easily reconfigurable and extendable. Thus, EvoBot provides a versatile and low cost tool to carry out research in multiple fields. We have in this paper described the overall implementation of the mechanical structure consisting of layers and modules and documented the performance of the robot, which are easily within the parameters required for a wide range of liquid-based experiments. In particular, a novel experiment with MFCs has been carried out, where the robot nurtures interactively an MFC based on its voltage. We hope that the unique features of this platform can form the basis for new lines of research in artificial life.

\section{Acknowledgments}

The authors would like to thank the E.U. Future and Emerging Technologies who supported this work through EVOBLISS grant no. 611640 and the members of the EVOBLISS consortium. In particular, Martin Hanczyc and Juan Manual Parrilla Gutiérrez, who were heavily involved in developing the concept.

\section{References}

Gajda, I., Greenman, J., Melhuish, C., and Ieropoulos, I. (2015). Simultaneous electricity generation and microbially-assisted electrosynthesis in ceramic mfcs. Bioelectrochemistry, 105:58-64.

Gutierrez, J. (2012). Automatic liquid handling for artificial life research. Master's thesis, University of Southern Denmark.

Gutierrez, J., Hinkley, T., Taylor, J., Yanev, K., and Cronin, L. (2014). Evolution of oil droplets in a chemorobotic platform. Nature Communications, 5:1-8.

Hanczyc, M., Parrilla, J., Nicholson, A., Yanev, K., and Stoy, K. (2015). Creating and maintaining chemical artificial life by robotic symbiosis. Artificial Life, 1(1):47-54.

Ieropoulos, I., Greenman, J., Melhuish, C., and Horsfield, I. (2010). Ecobot-iii: a robot with guts. In International Conference on the Synthesis and Simulation of Living Systems (ALIFE).

Kong, F., Yuan, L., Zheng, Y., and Chen, W. (2012). Automatic liquid handling for life science: A critical review of the current state of the art. Journal of Laboratory Automation, 17(3):169-185.

Müller, A., Amaldass, A., Yanev, K., and Rasmussen, S. (2015). Do it yourself (diy) liquid handling robot for evolutionary search exploration. In European Conference on Artificial Life.

Nejatimoharrami, F., Stoy, K., and Faina, A. (2016). An open-source, low-cost robot for performing reactive liquid handling experiments. In Society for Laboratory Automation and Screening (SLAS 2016).

Yim, M., Shen, W.-M., Salemi, B., Rus, D., Moll, M., Lipson, H., Klavins, E., and Chirikjian, G. (2007). Modular self-reconfigurable robot systems. In IEEE Robotics \& Automation Magazine, pages 43-52. 